\title{
A flux loop mechanism in two-dimensional stratified turbulence
}

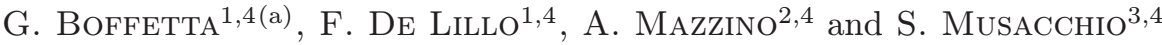 \\ ${ }^{1}$ Dipartimento di Fisica Generale and INFN, Università di Torino - via P. Giuria 1, 10125 Torino, Italy, EU \\ ${ }^{2}$ Dipartimento di Fisica and INFN, Università di Genova - via Dodecaneso 33, 16146 Genova, Italy, EU \\ ${ }^{3}$ CNRS, Laboratoire J.A. Dieudonné UMR 6621 - Parc Valrose, 06108 Nice, France, EU \\ ${ }^{4}$ International Collaboration on Turbulence Research
}

received 12 April 2011; accepted in final form 10 June 2011

published online 7 July 2011

PACS 47.27.E- - Turbulence simulation and modeling

PACS 47.55.Hd - Stratified flows

\begin{abstract}
We discuss the phenomenology of energy transfer in two-dimensional, weakly stably stratified turbulence. Kinetic energy, mechanically injected at small scales, is transferred by turbulence towards large scales. This inverse cascade proceeds up to the Ozmidov scale, where buoyancy forces become effective. Kinetic energy is converted into potential energy, which is transferred back towards small scales via a turbulent cascade of density fluctuations. The resulting "flux loop" is a novel mechanism which produces a non-trivial stationary state in two-dimensional turbulence in the absence of a large-scale dissipation.
\end{abstract}

Copyright (C) EPLA, 2011

Introduction. - Stably stratified flows are common in many instances ranging from geophysical flows to astrophysical applications (for a review see, e.g., $[1,2]$ ). From a fundamental point of view, the consequences of stable stratification on turbulence and its effect on the energy cascade in three-dimensional flows have been deeply investigated by means of numerical simulations (see, e.g., [3-6]). In particular it has been found that stratification can induce a reduction of dimensionality which generates an energy transfer toward large-scale structures with a mechanism which is further enhanced by the presence of rotation (see, e.g., $[7,8]$ ).

In this letter we study the effects of stratification on the inverse cascade of two-dimensional turbulence. In particular, we consider a two-dimensional flow stably stratified in the vertical direction and we address the phenomenology originated by the interplay between hydrodynamic and buoyancy forces. This choice is motivated by experiments recently carried out on stratified soap films [9-11] which call for a deep investigation of physical mechanisms acting in this context. Previous numerical studies of twodimensional vertically stratified flows have mostly focused on regimes which share common features with the threedimensional case, such as the strongly stratified regime [12] or the development of instabilities [13]. Here we are interested in the effects of stable stratification on the fate of the inverse energy cascade, a process which is peculiar of

(a) E-mail: boffetta@to.infn.it two-dimensional turbulence. We recall that another interesting regime arises in the presence of unstable stratification which induces turbulent motion with Bolgiano scaling for velocity and temperature fluctuations [14,15].

We consider a two-dimensional fluid stably stratified in the vertical direction by a mean gradient in the density field $\bar{\rho}(y)=\rho_{0}-\gamma y$ in the gravitational field $\mathbf{g}=(0,-g)$. Within the Boussinesq approximation, the density field can be equivalently thought as a temperature field, since temperature and density are related via the thermal expansion coefficient $\beta$. Introducing the Brunt-Väisälä frequency $N=\left(\gamma g / \rho_{0}\right)^{1 / 2}$, the Boussinesq equations for the incompressible velocity field $\mathbf{u}=\left(u_{1}, u_{2}\right)$ and density fluctuations $\phi=\frac{1}{\gamma}(\rho-\bar{\rho}) \mathrm{read}$

$$
\begin{aligned}
& \partial_{t} \boldsymbol{u}+\boldsymbol{u} \cdot \nabla \boldsymbol{u}=-\nabla p+\nu \Delta \boldsymbol{u}-N^{2} \phi \hat{e}_{2}+\boldsymbol{f}, \\
& \partial_{t} \phi+\boldsymbol{u} \cdot \nabla \phi=\kappa \Delta \phi+u_{2},
\end{aligned}
$$

where $\nu$ and $\kappa$ represent molecular viscosity and diffusivity, respectively, and $\boldsymbol{f}$ represents an external mechanical force.

In the inviscid, unforced limit (1), (2) conserve the total energy (kinetic plus potential),

$$
E=E_{K}+E_{P}=\frac{1}{2}\left\langle u^{2}\right\rangle+\frac{1}{2} N^{2}\left\langle\phi^{2}\right\rangle .
$$

In the presence of forcing and dissipations, the energy input is $\varepsilon_{I}=\langle\boldsymbol{v} \cdot \boldsymbol{f}\rangle$ which, together with viscous and 
diffusive dissipations, gives the energy balance $\frac{\mathrm{d} E}{\mathrm{~d} t}=\varepsilon_{I}-$ $\varepsilon_{\nu}-\varepsilon_{\kappa}$.

In the limit $N \rightarrow 0$, eq. (1) reduces to usual twodimensional Navier-Stokes equations coupled with the transport equation for the passive scalar field $\phi$. In this case (1) has two inviscid conserved quantities: kinetic energy $E_{K}$ and enstrophy $Z=(1 / 2)\left\langle(\boldsymbol{\nabla} \times \boldsymbol{u})^{2}\right\rangle$ and the turbulent flow displays the Batchelor-Kraichnan phenomenology with a direct cascade of enstrophy and an inverse cascade of energy to large scales [16-18]. The scalar field $\phi$ is forced at large scales by the last term in (2) and develops a direct cascade transported by the incompressible velocity field.

When $N>0$ the situation is in principle very different as the coupled system (1), (2) has the single inviscid conserved quantity (3) and the usual arguments supporting an inverse energy cascade cannot be a priori applied. Nonetheless, the coupling between potential and kinetic energy is not effective at all scales. The buoyancy force $N^{2} \phi$ balances the inertial force in (1) at the Ozmidov (or Bolgiano) scale $L_{O}$. At scales much smaller than $L_{O}$ the buoyancy term in (1) is negligible with respect to the other terms and scalar fluctuations can be considered effectively passive [19].

In this letter we focus on the case of weakly stratified flows, in which the Ozmidov scale is smaller than the external scale of the system (the scale of the box) $L_{b o x}$ and larger than the forcing scale $\ell_{f}$, i.e. $\ell_{f}<L_{O}<L_{b o x}$, a regime in which we expect the following scenario. Kinetic energy is injected, by definition, at scale $\ell_{f}$ where buoyancy forces are negligible, and therefore transferred toward larger scales by an inverse cascade à la Kraichnan. When the cascade reaches the Ozmidov scale $L_{O}$ kinetic energy is converted in potential energy (density fluctuations) by the coupling terms in (1), (2). Density fluctuations are then transported passively toward small scales by a direct cascade and finally dissipated by diffusivity. Therefore, we conjecture that the system (1), (2) can reach a statistical stationary state even in the absence of a large-scale dissipation mechanism thanks to the "flux loop" mechanism described above (and shown in fig. 3) which converts the inverse flux of kinetic energy in the direct flux of potential energy.

The above argument can be made more quantitative using dimensional analysis. In the range of scales $\ell_{f} \ll \ell \ll$ $L_{O}$ we assume to have an inverse cascade of kinetic energy with constant flux $\varepsilon=\varepsilon_{I}-\varepsilon_{\nu} \simeq \delta_{\ell} u^{3} / \ell$ and therefore with Kolmogorov scaling for velocity increments,

$$
\delta_{\ell} u \simeq \varepsilon^{1 / 3} \ell^{1 / 3} .
$$

At the Ozmidov scale, kinetic energy flux is converted into potential energy which generates a direct cascade with flux $\varepsilon_{\phi} \simeq\left(\delta_{\ell} u / \ell\right) \delta_{\ell} \phi^{2}$. If the conversion is complete, i.e. if $\varepsilon=N^{2} \varepsilon_{\phi}$, one has for the density fluctuations

$$
\delta_{\ell} \phi \simeq N^{-1} \varepsilon^{1 / 3} \ell^{1 / 3}
$$

The Ozmidov scale is determined by balancing inertial and buoyancy terms in (1). Using (4) and (5) one obtains

$$
L_{O} \simeq N^{-3 / 2} \varepsilon^{1 / 2} .
$$

The inverse energy cascade is therefore halted at the scale $L_{O}$ with a kinetic energy $E_{K} \simeq\left(\delta_{L_{O}} u\right)^{2} \simeq \varepsilon / N$. This stationary state is reached with a characteristic time given by the inverse of the Brunt-Väisälä frequency. Indeed, when the integral scale $L(t)$ of turbulent fluctuations is still smaller than $L_{O}$, the input of density fluctuations is given by $\varepsilon_{\phi} \simeq \delta_{L} u \delta_{L} \phi$. Using (4) and (5) this gives $\varepsilon_{\phi} \simeq N^{-1} \varepsilon^{2 / 3} L^{2 / 3} \simeq N^{-1} E_{K}$. From the energy balance, assuming that $\varepsilon_{\kappa}=N^{2} \varepsilon_{\phi}$ (direct cascade) and that total energy is dominated by the kinetic component so that $\varepsilon_{\phi} \simeq N^{-1} E$, we have

$$
\frac{\mathrm{d} E}{\mathrm{~d} t}=\varepsilon-N E
$$

which gives

$$
E(t)=\frac{\varepsilon}{N}\left(1-e^{-N t}\right)
$$

The above arguments assume that $L_{O} \gg \ell_{f}$ and therefore they are valid for $N \ll \varepsilon^{1 / 3} \ell_{f}^{-2 / 3}$. For larger values of the Brunt-Väisälä frequency, stratification prevents the formation of the turbulent inverse cascade and energy injected into the system is immediately converted into potential energy. This regime of strong stratification has been investigated in [12], and displays a different phenomenology characterized by the transfer of energy toward vertical shear modes. Moreover, energy conversion is possible only if $L_{O} \leqslant L_{b o x}$ and therefore the condition $N \geqslant \varepsilon^{1 / 3} L_{b o x}^{-2 / 3}$ is necessary to reach a stationary state without accumulation of energy at the largest scale.

Of course, the effectiveness of the flux loop to stop the inverse cascade at $L_{O}$ and to produce a stationary state cannot be guaranteed a priori, as it depends on the detailed coupling between backward kinetic energy flux and forward potential energy flux. Therefore, one has to make use of laboratory experiments or numerical simulations in order to validate the theoretical scenario.

In this letter we report the results of a set of direct numerical simulations of eqs. (1), (2) on a square domain of size $L_{b o x}=2 \pi$ with periodic boundary conditions for different values of $N$. The simulations have been performed with a pseudospectral code, with $2 / 3$-dealiasing rule, at resolution $2048^{2}$. The flow is sustained by a Gaussian forcing $\boldsymbol{f}=\left(\partial_{y} \varphi,-\partial_{x} \varphi\right)$ with correlation function $\left\langle\varphi(\boldsymbol{x}, t), \varphi\left(\boldsymbol{x}^{\prime}, t^{\prime}\right)\right\rangle=\Phi \delta\left(t-t^{\prime}\right) \exp \left(-\left(\left|\boldsymbol{x}-\boldsymbol{x}^{\prime}\right| / \ell_{f}\right)^{2}\right)$. Parameters of the simulations are reported in the caption of fig. 1 where we show a snapshot of the density fluctuation field $\phi$ obtained in our simulations. In spite of the stratification in the vertical direction, the density fluctuations are almost isotropic. Also the turbulent flow does not show any significant anisotropy at scales within the inertial range. The ratio between the rms velocities in the vertical and horizontal direction measured in our 


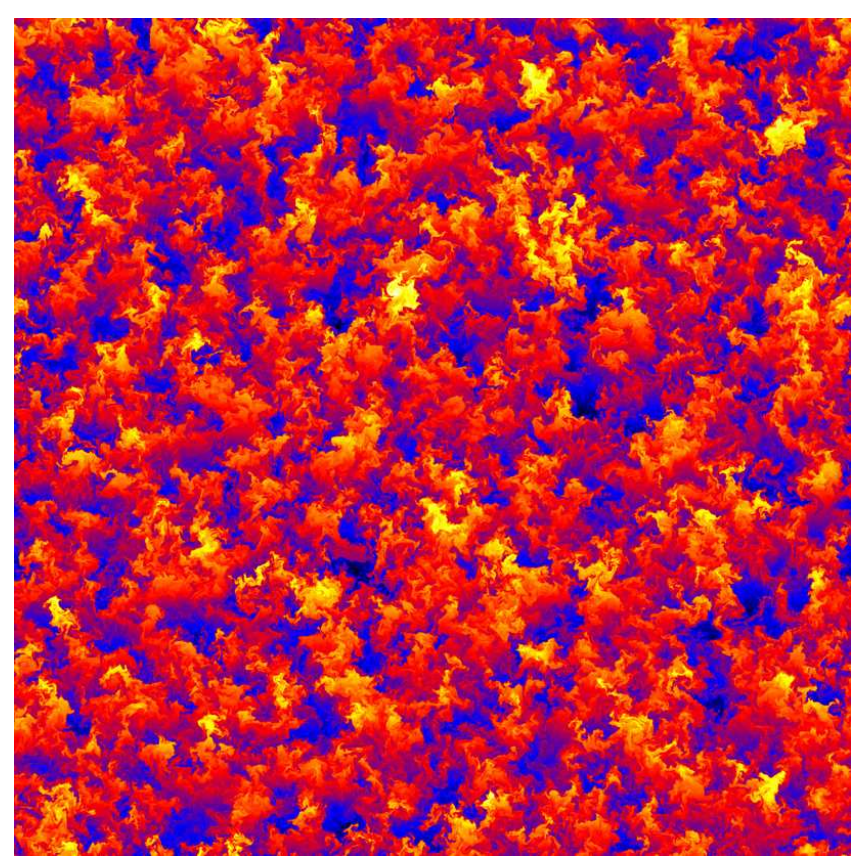

Fig. 1: (Colour on-line) Snapshot of the density fluctuation field obtained in numerical simulation with parameters $\nu=1 \times$ $10^{-5}, \kappa=4 \times 10^{-5}, \ell_{f}=\pi / 128, \varepsilon_{I}=3.8 \cdot 10^{-3}$, and $N^{2}=1 / 4$.

simulations varies in the range $0.95-0.97$, decreasing monotonically as the stratification is increased. These results are in striking contrast with the strong anisotropy of the density and velocity fields observed in the regime of strong stratification, i.e. when $L_{0}<\ell_{f}$, both in twoand three-dimensional flows $[3-6,12]$. Indeed, the lack of anisotropy in the $2 \mathrm{D}$ weakly stratified case is in agreement with the results reported in [12], and gives a first indication that the upscale energy transfer is arrested at the Ozmidov scale, thus preventing the formation of large anisotropic structures. We have verified that a similar behavior appears also in numerical simulations of two-dimensional Navier-Stokes equation (without stratification) in the presence of an anisotropic friction term (i.e. active only on the vertical velocity). It is worth mentioning that a similar phenomenology has been observed in the case of the stratified two-dimensional Kolmogorov flow [13].

The results of our simulations indicates that the flux loop mechanism depicted above is effective to stop the inverse energy cascade at the Ozmidov scale and allows to reach a steady state even in the absence of large-scale dissipation. As it is shown in fig. 2 (panels (a) and (b)), in the stratified cases both the kinetic and potential energy attain constant values as the system evolves starting from the $\mathbf{u}=0, \phi=0$ initial condition. This situation differs from the test case without stratification $\left(N^{2}=0\right)$ in which $E_{K}$ grows linearly in time, with the growth rate prescribed by the energy balance $\frac{\mathrm{d} E_{K}}{\mathrm{~d} t}=\varepsilon_{I}-\varepsilon_{\nu}$. Consistently, we observe that in the stratified cases the energy growth rate $\varepsilon_{I}-\varepsilon_{\nu}-\varepsilon_{\kappa}$ (shown in fig. 2, panel (c)) decreases exponentially, with a decay rate which is proportional to
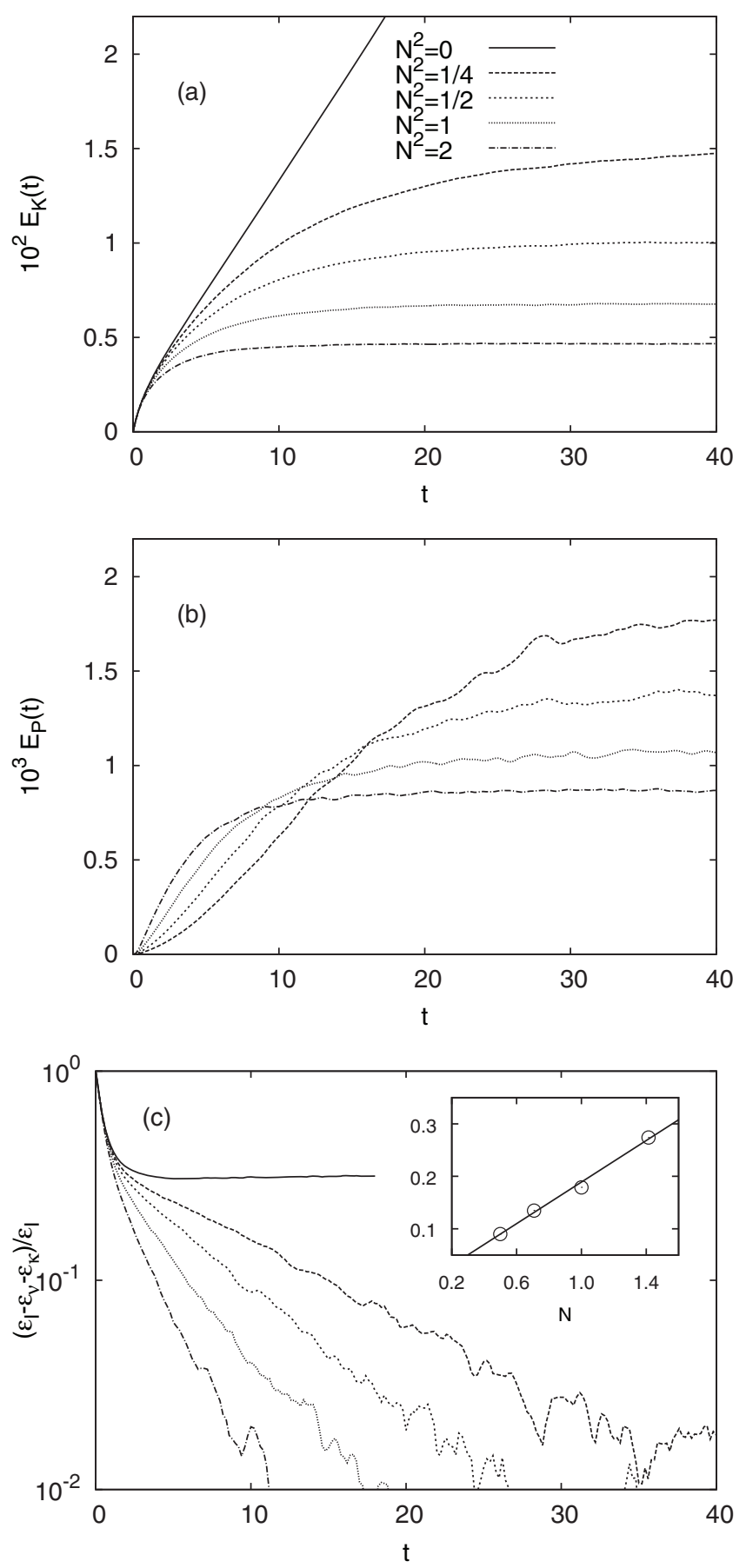

Fig. 2: Temporal evolution of kinetic energy $E_{K}$ (panel (a)) and potential energy $E_{P}$ (panel (b)) and energy balance (panel (c)) for various $N^{2}$. The inset of panel (c) shows the rate of exponential decay of energy growth rate as a function of $N$. The line represent the linear best fit.

$N$, as predicted by (8). The ratio between $E_{P}$ and $E_{K}$ measured in our simulations is an increasing function of $N$ and varies in the range $0.11-0.19$, therefore $E_{P} \ll E_{K}$ as assumed in the derivation of (8).

The kinetic and potential energy spectra measured in the statistically steady state (reported in fig. 3) have a maximum close to the Ozmidov scale $L_{O}$, where the 


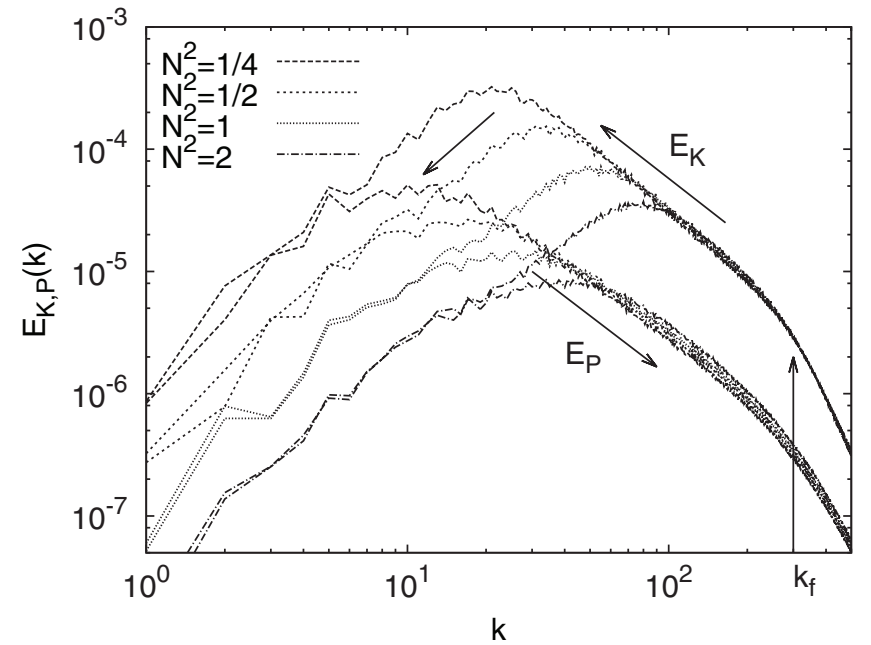

Fig. 3: Kinetic and potential energy spectra for various $N^{2}$. The arrows indicate the "flux loop".

inverse cascade of $E_{K}$ is stopped. More precisely, the maximum of the kinetic energy spectrum is attained at a wave number slightly larger (about a factor 2) than that of the potential energy spectrum. At high wave numbers, $k \gg L_{O}^{-1}$, the spectra display an inertial range close to Kolmogorov-Obukhov scaling $E_{K, P} \sim k^{-5 / 3}$, in agreement with the hypothesis that the buoyancy effects become almost negligible at scales $\ell \ll L_{O}$. However, a weak subdominant effect of the buoyancy force, which pumps the direct cascade of density fluctuation in distributed way, can be detected in the slope of the potential energy spectrum, which is slightly flatter than Obukhov scaling. At small wave numbers $k \ll L_{O}^{-1}$ the spectra of kinetic and potential energy have the same amplitude, and are characterized by a scaling $E_{K, P} \sim k^{2}$.

In fig. 4 we show the collapse of the maximum of the kinetic, potential and exchange energy spectra, the latter being defined as $E_{X}(k)=N / 2 \int_{|\boldsymbol{q}|=k} \mathrm{~d}^{2} \boldsymbol{q}\left[u_{2}(\boldsymbol{q}) \phi(-\boldsymbol{q})+\right.$ c.c.], for various stratification, according to the dimensional prediction $E_{K, P, X}\left(k L_{O}\right) \simeq L_{O} \varepsilon / N$. We note that the dimensional scaling is less accurate for $E_{P}(k)$ than $E_{K}(k)$. As we will discuss, this is probably due to the contamination of the dissipative range. The exchange energy spectra have a maximum close to $k \sim L_{O}^{-1}$, and display a power-law behavior for $k \ll L_{O}^{-1}$ and $k \gg L_{O}^{-1}$, showing that the exchange between kinetic and potential energy occurs at all scales, and is maximum at the Ozmidov scale.

The flux loop mechanism which allows for the formation of a stationary state is understood in terms of kinetic and potential energy fluxes. From (1), (2) we can write

$$
\begin{aligned}
& \frac{\mathrm{d}}{\mathrm{d} t} \int_{k}^{\infty} E_{K}(q) \mathrm{d} q=\Pi_{K}(k)+\Pi_{X}(k)-D_{\nu}(k)+F(k), \\
& \frac{\mathrm{d}}{\mathrm{d} t} \int_{k}^{\infty} E_{P}(q) \mathrm{d} q=\Pi_{P}(k)-\Pi_{X}(k)-D_{\kappa}(k),
\end{aligned}
$$

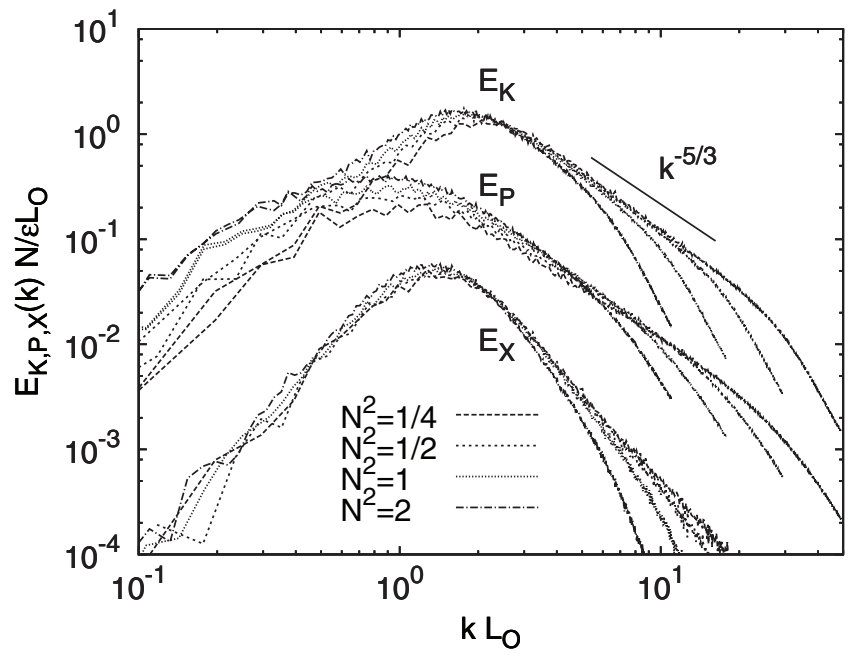

Fig. 4: Kinetic, potential and exchange energy spectra for various $N^{2}$ rescaled at the Ozmidov scale $L_{O}$. The exchange energy spectra $E_{X}(k)$ are multiplied by a factor $10^{-1}$ for plotting purposes.

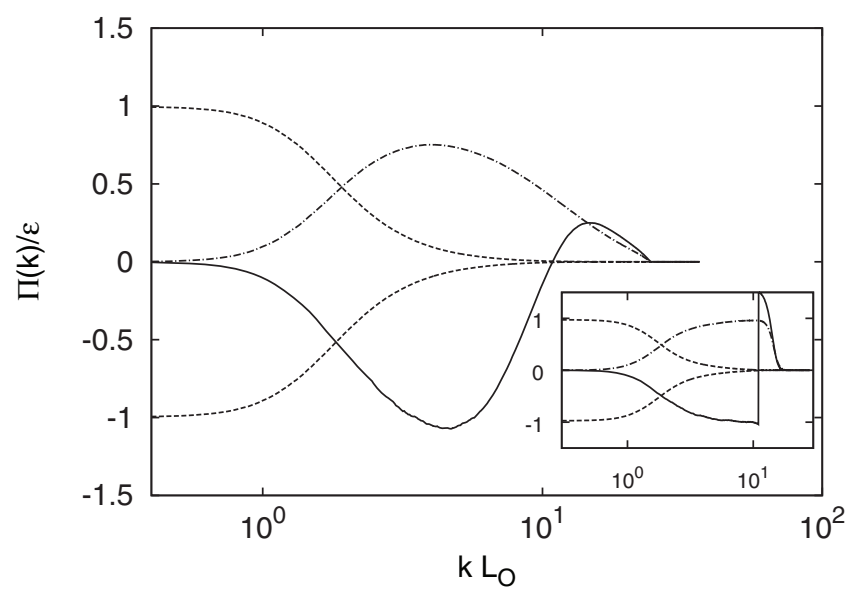

Fig. 5: Fluxes of kinetic energy $\Pi_{K}(k)$ (solid line), potential energy $\Pi_{P}(k)$ (dash-dotted line), and exchange integrals $\pm \Pi_{X}(k)$ (dashed lines) normalized with the energy input rate. Here $N^{2}=1 / 4$. Inset: same picture for a simulation with hyperviscosity and narrow-banded forcing.

where $\Pi_{K}(k)$ and $\Pi_{P}(k)$ are the kinetic and potential energy fluxes, $\Pi_{X}(k)=N^{2} / 2 \int_{|\boldsymbol{q}| \geqslant k} \mathrm{~d}^{2} \boldsymbol{q}\left[u_{2}(\boldsymbol{q}) \phi(-\boldsymbol{q})+\right.$ c.c. $]$ is the exchange integral, $D_{\nu}(k)$ and $D_{\kappa}(k)$ are the dissipative contributions and $F(k)$ is the contribution by the forcing. The behavior of the $\Pi_{K}(k), \Pi_{P}(k)$ and $\Pi_{X}(k)$ terms is shown in fig. 5 . The negative flux of kinetic energy vanishes at wave numbers smaller than $L_{O}^{-1}$, where the conversion from kinetic to potential energy takes place and the exchange integral $\Pi_{X}(k)$ is different from zero. This is then transferred toward small scales via a direct cascade with positive flux.

Let us notice that in our simulation with standard dissipation, i.e. Newtonian viscosity and diffusivity, the dissipative effects are not negligible at wave numbers 

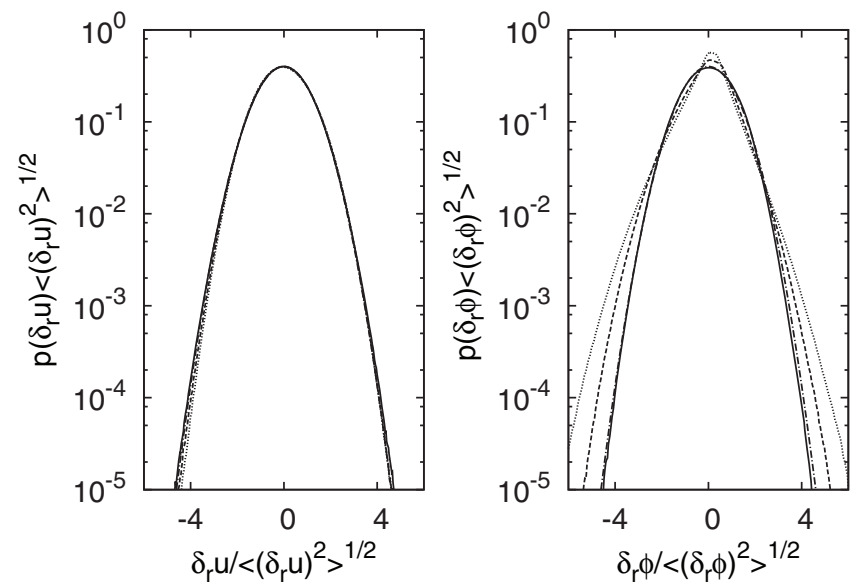

Fig. 6: PDFs of longitudinal velocity increments $\delta_{r} u$ (left panel) and scalar increments $\delta_{r} \phi$ (right panel) at scales $r / L_{b o x}=1 / 32$ (solid line), $r / L_{b o x}=1 / 128$ (dashed line) and $r / L_{b o x}=1 / 256$ (dotted line). Here $N^{2}=1 / 2$. The dash-dotted line represents a standard Gaussian.

corresponding to the turbulent-cascade range. Such contamination is clearly visible in the fluxes which do not display a clear scale-independent plateau. Further, the positive peak of the flux $\Pi_{P}$ does not balance exactly the negative peak of the flux $\Pi_{K}$, because a part of energy is removed by dissipation at any scales along the turbulent cascade. As a consequence, the actual value of the flux of potential energy is smaller than the asymptotic value $\varepsilon$, and depends on $N$. This also explains the slight deviation observed in the collapse of the potential energy spectra. Such spurious effects are expected to disappear in the limit of vanishing dissipation. To check this we have performed a test simulation with narrow-banded forcing and hyperdissipation, which allows to confine the dissipative effects at high wave numbers. In this case the fluxes of $E_{K}$ and $E_{P}$ perfectly balance each other (see inset of fig. 5).

It is interesting to note that this corresponds to a state in which the net flux of the inviscid invariant $E=E_{P}+E_{K}$ is zero at all scales $\ell \leqslant \ell_{f}$. The occurrence of such zero-flux state is highly non-trivial, since it results from the cancellations of two cascade processes which have substantially different properties. In particular, the probability distribution functions (PDFs) of longitudinal velocity increments $\delta_{r} u$, computed at scales $r$ within the inertial range, are self-similar and almost Gaussian (see left panel of fig. 6), showing that the inverse energy cascade of $E_{K}$ is not intermittent, as it is observed in the absence of stratification [18]. On the contrary, the PDFs of scalar increments $\delta_{r} \phi$ (shown in the right panel of fig. 6) reveal that the direct cascade of potential energy $E_{P}$ is characterized by the presence of small-scale intermittency, similar to those observed in the case of a passive scalar field. Therefore, the zero-flux state for the total energy $E$ is originated by the coexistence of a non-intermittent cascade of $E_{K}$ and an intermittent cascade of $E_{P}$ which proceed in opposite directions.
It is also worth noting that the conversion of kinetic energy into potential energy is not confined in a narrow range of scales close to the Ozmidov scale. On the contrary, it takes place in a broad range of scales (more than one decades), as is shown both by the power-law tails of the exchange energy spectrum $E_{X}(k)$ (see fig. 4) and by the slow convergence of the exchange integral to the asymptotic value $\Pi_{X}(k) \rightarrow \varepsilon=\varepsilon_{I}-\varepsilon_{\nu}$ for $k L_{O} \ll 1$. Such convergence, which is required to obtain a steady state, may not be completely achieved if the stratification is reduced so much that the Ozmidov scale $L_{O}$ gets close to the scale of the box $L_{b o x}$. In this case, one may argue that the excess of kinetic energy which is not completely converted into potential energy could accumulate at the scale $L_{O}$, leading to the formation of a condensate state at wave number $L_{O}^{-1}$. This scenario is consistent with the results reported in [12], which have shown the possibility to find unsteady states characterized by the presence of strong vortices at the scale $\ell_{0}$ in the weakly stratified case.

In conclusion, in this letter we have discussed a simple mechanism, based on a flux loop process taking place in wave number space, which is responsible for the formation of non-trivial stationary states in stably stratified twodimensional turbulence. The existence of such states has been confirmed by the results of our numerical simulations performed within the framework of the Boussinesq approximation. The mechanism depicted here is consistent with previous reports of the arrest of the inverse energy cascade caused by stratification [13], and does not exclude a priori the possibility to observe unsteady condensed states populated by strong vortical structures [12]. The joint efforts of numerical studies and laboratories experiments with soap films could help to achieve a better understanding of the conditions which could lead to the emergence of these condensed states and their influences on the upscale energy transfer.

We thank the Cineca Supercomputing Center (Bologna, Italy) for the allocation of computational resources. GB and AM acknowledge the hospitality of the Turbulence program at KITP where part of the results have been obtained.

\section{REFERENCES}

[1] Hopfinger E. J., J. Geophys. Res., 92 (1987) 5287.

[2] Riley J. J. and Lelong M.-P., Annu. Rev. Fluid Mech., $32(2000) 613$.

[3] Herring J. R. and Métais O., J. Fluid Mech., 202 (1989) 97.

[4] Waite M. L. and Bartello P., J. Fluid Mech., 517 (2004) 281.

[5] Lindborg E., J. Fluid Mech., 550 (2006) 207. 
[6] Brethouwer G., Brillant P., Lindborg E. and Chomaz J.-M., J. Fluid Mech., 585 (2007) 343.

[7] Smith L. M. and Waleffe F., J. Fluid Mech., 451 (2002) 145.

[8] Métais O., Bartello P., Garnier E., Riley J. J. and Lesieur M., Dyn. Atmos. Oceans, 23 (1996) 193.

[9] Zhang J., Wu X. L. and Xia K.-Q., Phys. Rev. Lett., 94 (2005) 174503.

[10] Seychelles F., Amarouchene Y., Bessafi M. and Kellay H., Phys. Rev. Lett., 100 (2008) 144501.

[11] Martin B. and Wu X. L., Phys. Rev. Lett., 80 (1998) 1892.

[12] Smith L. M., Numerical Study of two-dimensional stratified turbulence, in Advances in Wave Interaction and
Turbulence, edited by Milewski P. A., Smith L. M., Waleffe F. and TABak E. G. (American Mathematical Society, Providence, RI) 2001, p. 91.

[13] Balmforth N. J. and Young Y.-N., J. Fluid Mech., 450 (2002) 131.

[14] Chertkov M., Phys. Rev. Lett., 91 (2003) 115001.

[15] Celani A., Mazzino A. and Vozella L., Phys. Rev. Lett., 96 (2006) 134504.

[16] Kraichnan R. H. and Montgomery D., Rep. Prog. Phys., 43 (1980) 547.

[17] Boffetta G., J. Fluid Mech., 589 (2007) 253.

[18] Boffetta G. and Musacchio S., Phys. Rev. E, 82 (2010) 016307.

[19] Celani A., Cencini M., Mazzino A. and Vergassola M., New J. Phys., 6 (2004) 72. 